\title{
Dissipativity, passivity and feedback passivity in the nonlinear discrete-time setting
}

Link to publication record in Manchester Research Explorer

\section{Citation for published version (APA):}

Navarro Lopez, E., Navarro-Lopez, E. M., \& Fossas-Colet, E. (2002). Dissipativity, passivity and feedback passivity in the nonlinear discrete-time setting. In 15th IFAC Triennial World Congress (Vol. 1, pp. 1-6). International Federation of Automatic Control (IFAC). http://www.cs.man.ac.uk/ navarroe/papers/enavarro_ifac02_1.pdf

\section{Published in:}

15th IFAC Triennial World Congress

\section{Citing this paper}

Please note that where the full-text provided on Manchester Research Explorer is the Author Accepted Manuscript or Proof version this may differ from the final Published version. If citing, it is advised that you check and use the publisher's definitive version.

\section{General rights}

Copyright and moral rights for the publications made accessible in the Research Explorer are retained by the authors and/or other copyright owners and it is a condition of accessing publications that users recognise and abide by the legal requirements associated with these rights.

\section{Takedown policy}

If you believe that this document breaches copyright please refer to the University of Manchester's Takedown Procedures [http://man.ac.uk/04Y6Bo] or contact uml.scholarlycommunications@manchester.ac.uk providing relevant details, so we can investigate your claim.

\section{OPEN ACCESS}




\title{
DISSIPATIVITY, PASSIVITY AND FEEDBACK PASSIVITY IN THE NONLINEAR DISCRETE-TIME SETTING
}

\author{
E.M. Navarro-López* E. Fossas-Colet ${ }^{*}$ \\ ${ }^{*}$ Universitat Politècnica de Catalunya \\ Institut d'Organització i Control \\ Avda. Diagonal 647, 11 planta, \\ 08028 Barcelona, Spain
}

\begin{abstract}
Necessary and sufficient conditions for a class of dissipative nonlinear multiinput multi-output discrete-time systems are given. The conditions existing in the literature, addressed as Kalman-Yakubovich-Popov conditions, for the dissipative, passive or lossless cases, are derived from the proposed dissipativity characterization. Feedback passivity is solved for a class of multi-input multi-output nonlinear discrete-time systems affine in the control input using the properties of the relative degree and zero dynamics of the non-passive system. This is the first solution presented in the literature to such a problem in the discrete-time setting. The passification methodology is illustrated by means of an example.
\end{abstract}

Keywords: Discrete-time systems, Nonlinear systems, Energy control, Passive elements, Feedback stabilization.

\section{INTRODUCTION. MOTIVATIONS}

The study of dissipativity-related concepts in the nonlinear discrete-time setting is an interesting field for which a lot of problems remain unsolved. Two main problems of utmost importance will be considered. On the one hand, the proposal for nonlinear non-affine in the input discrete-time systems of what is called Kalman-Yakubovich-Popov (KYP) conditions, that is, necessary and sufficient conditions for the characterization of a passive or a dissipative system. On the other hand, the passification problem or the problem of rendering a system passive by means of a static feedback control law will be presented.

The characterization of passive discrete-time systems is given in the literature for linear systems (Hitz and Anderson, 1969), and for nonlinear systems affine in the control input (Byrnes and Lin, 1993; Lin and Byrnes, 1995). Lossless systems affine in the control input are also characterized (Byrnes and Lin, 1994;
Sengör, 1995). Necessary and sufficient conditions for dissipative systems affine in the control input are presented in (Sengör, 1995); the linear case is treated in (Goodwin and Sin, 1984). In both cases, the supply function takes the form $s(y, u)=y^{T} Q y+2 y^{T} S u+$ $u^{T} R u$. For multi-input multi-output passive systems which are nonlinear in the states and the control input there are only necessary conditions for passivity (Lin, 1995). For single-input multi-output nonlinear non-affine in the input systems, the KYP conditions are proposed in (Monaco and Normand-Cyrot, 1997) where the authors phocus on systems which can be expanded by exponential Lie series.

The problem of the establishment of conditions for a nonlinear discrete-time system to be rendered passive or dissipative via a state feedback has not been solved yet. This problem has only been solved for lossless systems (Byrnes and Lin, 1994). The problem of feedback dissipativity for nonlinear discrete-time systems 
has been solved in a non-general manner in (Navarro et al., 2001a, b).

The study of the properties of the relative degree and the zero dynamics of a passive system has played an important role in understanding problems such as feedback passivity or the stabilization of passive systems in the continuous-time setting, see (Byrnes et al., 1991). For general discrete-time systems, the implications of dissipativity and passivity in the relative degree and the zero dynamics have not been established yet, they have only been studied for the losslessness case, see (Byrnes and Lin, 1994). In this paper, the properties of the relative degree and the zero dynamics of a passive system will be related to its feedback passivity property.

The paper is organized as follows. Section (2) presents necessary and sufficient conditions for the characterization of a class of dissipative nonlinear discrete-time systems. Section (3) revisits the properties of the zero dynamics of passive systems. Section (4) presents a solution to the passification problem for a class of nonlinear discrete-time systems through the relative degree and the zero dynamics properties. Conclusions and suggestions for further research are given in the last section.

\section{DISSIPATIVITY IN DISCRETE-TIME SYSTEMS UNDER STUDY}

Let a system of the form,

$$
\begin{aligned}
x(k+1) & =f(x(k), u(k)), x \in \mathscr{X}, u \in \mathscr{U} \\
y(k) & =h(x(k), u(k)), y \in \mathscr{Y}
\end{aligned}
$$

where $f: \mathscr{X} \times \mathscr{U} \rightarrow \mathscr{X}$ and $h: \mathscr{X} \times \mathscr{U} \rightarrow \mathscr{Y}$ are smooth maps, with $\mathscr{X} \subset \mathfrak{R}^{n}, \mathscr{U}, \mathscr{Y} \subset \mathfrak{R}^{m}, k \in \mathscr{Z}_{+}:=$ $\{0,1,2, \ldots\}$. All considerations are restricted to an open set of $\mathscr{X} \times \mathscr{U}$ containing $\left(x^{*}, u^{*}\right)$, having $x^{*}=$ $f\left(x^{*}, u^{*}\right)$. There is no loss of generality in considering $\left(x^{*}, u^{*}\right)=(0,0)$ and $h(0,0)=0$, from now on they both are considered.

Definition 1. (Byrnes and Lin, 1994) A system of the form (1) with a properly chosen output (2) is said to be dissipative with respect to a supply rate function $s: \mathscr{Y} \times \mathscr{U} \rightarrow \mathfrak{R}$ if there exists a positive definite function $V: \mathscr{X} \rightarrow \mathfrak{R}^{+}, V(0)=0$, called the storage function, such that

$$
\begin{aligned}
& V(x(k+1))-V(x(k)) \leq s(y(k), u(k)), \\
& \forall(x(k), u(k)) \in \mathscr{X} \times \mathscr{U}, \forall k
\end{aligned}
$$

This system will be also referred as $V$-dissipative.

Definition 2. System (1)-(2) is said to be passive if it is dissipative with respect to the supply rate $s(y(k), u(k))=y^{T}(k) u(k)$.
Definition (1) is rewritten in the following way.

Definition 3. A system of the form (1) with a properly chosen output (2) is said to be dissipative with respect to a supply rate function $s$ if there exist a positive definite function $V, V(0)=0$, and a function $\phi(\cdot, u)$ which is positive semi-definite for each $u \in \mathscr{U}$ such that

$$
\begin{aligned}
& V(x(k+1))-V(x(k))=s(h(x(k), u(k)), u(k))- \\
& -\phi(x(k), u(k)), \forall(x(k), u(k)) \in \mathscr{X} \times \mathscr{U}, \forall k
\end{aligned}
$$

The function $\phi$ is regarded as the dissipation rate function (Hill and Moylan, 1980).

Definition 4. System (1)-(2) is said to be lossless if the dissipation rate function is zero.

Theorem 5. Suppose there exists a $\mathscr{C}^{2}$ function $V$ such that $V(f(x, u))$ is quadratic in $u$. Then, a discretetime nonlinear system of the form (1)-(2) is $V$ dissipative with a $\mathscr{C}^{2}$ supply function $s(y, u)$, satisfying $s(h(x, u), u)$ quadratic in $u$, and with storage function $V, V: \mathscr{X} \rightarrow \mathfrak{R}^{+}, V(0)=0$, if and only if, there exist real functions $l(x), m(x)$ and $k(x)$, all of appropriate dimensions such that

$$
\begin{aligned}
V(f(x, 0))-V(x)= & s(h(x, 0), 0)-l^{T}(x) l(x)- \\
& -m^{T}(x) m(x)
\end{aligned}
$$

$$
\begin{aligned}
\left.\frac{\partial V(\alpha)}{\partial \alpha}\right|_{\alpha=f(x, 0)} & \left.\frac{\partial f}{\partial u}\right|_{u=0}+2 l^{T}(x) k(x)= \\
= & \left.\frac{\partial s(y, u)}{\partial u}\right|_{u=0}+\left.\frac{\partial s}{\partial y} \frac{\partial h}{\partial u}\right|_{u=0}
\end{aligned}
$$

$$
\begin{aligned}
\left.\left(\frac{\partial f}{\partial u}\right)^{T}\right|_{u=0} & \left.\left.\frac{\partial^{2} V(\alpha)}{\partial \alpha^{2}}\right|_{\alpha=f(x, 0)} \frac{\partial f}{\partial u}\right|_{u=0}+ \\
& +\left.\left.\frac{\partial V(\alpha)}{\partial \alpha}\right|_{\alpha=f(x, 0)} \frac{\partial^{2} f}{\partial u^{2}}\right|_{u=0}= \\
& =\left.\frac{\partial^{2} s(h(x, u), u)}{\partial u^{2}}\right|_{u=0}-2 k^{T}(x) k(x)
\end{aligned}
$$

with

$$
\begin{aligned}
\frac{\partial^{2} s(h(x, u), u)}{\partial u^{2}} & =\frac{\partial^{2} s(y, u)}{\partial u^{2}}+ \\
& +\frac{\partial s}{\partial y \partial u} \frac{\partial h}{\partial u}+\frac{\partial s}{\partial y} \frac{\partial^{2} h}{\partial u^{2}}
\end{aligned}
$$

Proof (Necessity): Consider the dissipativity definition (3). If system (1)-(2) is $V$-dissipative, there exists $\phi$ satisfying (4). Since $V(f(x, u))$ and $s(h(x, u), u)$ are 
quadratic in $u$, the dissipation rate function $\phi$ can be written as follows

$$
\begin{aligned}
\phi(x, u) & =[l(x)+k(x) u]^{T}[l(x)+k(x) u]+ \\
& +m^{T}(x) m(x) \geq 0, \forall u \in \mathscr{U}
\end{aligned}
$$

for some real functions $m(x), l(x)$ and $k(x)$. Condition (5) is obtained restricting (4) to $u=0$, and taking $\phi(x, u)$ as defined in (8). Conditions (6) and (7) follow from the first-order derivative and the second-order derivative of (4) with respect to $u$, considering (8) and $u=0$.

(Sufficiency): Assume there exist real functions $m(x)$, $l(x), k(x)$ which satisfy conditions (5)-(7). Multiplying equality (6) by $u^{T}$ from the left and adding (5), it is obtained

$$
\begin{aligned}
& V(f(x, 0))-V(x)+\left.u^{T} \frac{\partial}{\partial u} V(f(x, u))\right|_{u=0}= \\
& =s(h(x, 0), 0)+\left.u^{T} \frac{\partial}{\partial u} s(h(x, u), u)\right|_{u=0}- \\
& -2 l^{T}(x) k(x) u-l^{T}(x) l(x)-m^{T}(x) m(x)
\end{aligned}
$$

Adding to the right-hand side term of (9) $u^{T} k^{T} k u$ $-u^{T} k^{T} k u$, and using (7), one yields

$$
\begin{aligned}
& V(f(x, 0))+\left.u^{T} \frac{\partial}{\partial u} V(f(x, u))\right|_{u=0}+ \\
& +\left.\frac{1}{2} u^{T} \frac{\partial^{2}}{\partial u^{2}} V(f(x, u))\right|_{u=0} u-V(x)= \\
& =s(h(x, 0), 0)+\left.u^{T} \frac{\partial}{\partial u} s(h(x, u), u)\right|_{u=0}+ \\
& +\left.\frac{1}{2} u^{T} \frac{\partial^{2}}{\partial u^{2}} s(h(x, u), u)\right|_{u=0} u-\phi(x, u),
\end{aligned}
$$

with $\phi$ given in (8). By claiming that $V(f(x, u))$ and $s(h(x, u), u)$ are quadratic in $u$, the second-order Taylor expansion at $u=0$ of $V(f(x, u))$ and $s(h(x, u), u)$ can be considered in (10), and (4) is obtained.

Remark 6. As it is recommended in (Sengör, 1995), a new function $m(x)$ has been considered, in comparison to the passivity conditions given in (Byrnes and Lin, 1993) for nonlinear discrete-time systems affine in the control input.

If $V(f(x, u))$ and $s(h(x, u), u)$ are not considered to be quadratic in $u$, conditions (5)-(7) yield only necessary conditions, as it is shown in (Navarro et al., 2001b).

Lossless systems of the form (1)-(2) can also be characterized by means of conditions (5)-(7), with $\phi(x, u)=0$, i.e., with $l(x)=k(x)=m(x)=0$.

From dissipativity characterization of Theorem (5) the existing KYP conditions appeared in the literature can be derived, either for the linear or the nonlinear case. Passivity conditions for nonlinear discrete- time which are affine in the control input appearing in (Byrnes and Lin, 1993) are obtained taking $s(y, u)=y^{T} u$ and $m(x)=0$, and losslessness conditions (Byrnes and Lin, 1994) with $s(y, u)=y^{T} u$ and $\phi(x, u)=l(x)=k(x)=m(x)=0$. In order to obtain dissipativity conditions for nonlinear affine in the control input systems presented in (Sengör, 1995), the left-hand side of equality (5) would be $V(f(x)-x)$ and in (6) and (7), $z=f(x)-x$ should be considered with $V(x)=B^{T}(x)+x^{T} C(x) x$, with $B$ and $C$ matrices of appropriate dimensions, and $s(y, u)=y^{T} Q y+$ $2 y^{T} S u+u^{T} R u$, with $Q, S, R$ constant matrices, $Q$ and $R$ symmetric. In order to obtain the losslessness conditions presented in this work, in addition, it must be considered $\phi(x, u)=k(x)=l(x)=m(x)=0$. For the linear case: passivity conditions appearing in (Hitz and Anderson, 1969) are obtained taking $m(x)=0$, $V(x)=\frac{1}{2} x^{T} P x, s(y, u)=y^{T} u$, and dissipativity conditions presented in (Goodwin and Sin, 1984) are obtained by taking $m(x)=0, s(y, u)=y^{T} Q y+2 y^{T} S u+$ $u^{T} R u, V(x)=\frac{1}{2} x^{T} P x$, with $P$ a real symmetric positive definite matrix. In these results, the systems are affine in the input; this results in supply functions quadratic in the input when the variables are the states and the input.

\section{ZERO DYNAMICS OF PASSIVE NONLINEAR DISCRETE-TIME SYSTEMS}

Assume

$$
\left.\frac{\partial h(x, u)}{\partial u}\right|_{\substack{x=0 \\ u=0}} \neq 0
$$

Then, by the implicit function theorem, there exists $u^{*}: \vartheta_{1} \rightarrow \mathscr{U}$ defined in a neighbourhood of $x=0$ such that $h\left(x, u^{*}\right)=0, \forall x \in \vartheta_{1}$. The zero dynamics of system (1)-(2) is defined by $f^{*}=f\left(x, u^{*}\right)$ where $\left(x, u^{*}\right) \in \mathscr{Z}^{*}=\left\{(x, u): x \in \vartheta_{1}, h(x, u)=0\right\}$.

Definition 7. A system of the form (1)-(2) has a locally passive zero dynamics if there exists a $\mathscr{C}^{2}$ positive definite function $V$, locally defined on the neighbourhood $\vartheta_{2}$ of $x=0$ in $\mathscr{X}$, s.t. $V(0)=0$ and

$$
V\left(f\left(x, u^{*}\right)\right) \leq V(x), \quad \forall x \in \vartheta_{2}
$$

Theorem 8. Suppose system (1)-(2) satisfying (11) to be passive with a storage function $V$ which is positive definite, and $V(0)=0$. Then, the zero dynamics of (1)(2) locally exists at $x=0$ and is passive.

Proof By (11), system (1)-(2) has relative degree 0 in a neighbourhood of $x=0$ and its zero dynamics, indeed, locally exists in a neighbourhood of $x=0$ in $\Re^{n}$. As (1)-(2) is passive, relation (3) is met with $s(y, u)=$ $y^{T} u$. Setting $u=u^{*}$ such that $y=h\left(x, u^{*}\right)=0$, one yields to $f^{*}(x)$. Since the zero dynamics is restricted to $\mathscr{Z}^{*}$, inequality (3) is converted into equation (12). 


\section{A SOLUTION TO THE PASSIFICATION PROBLEM}

The passification problem will be studied for a class of nonlinear discrete-time systems linear in the input. Let the system,

$$
\begin{aligned}
x(k+1) & =f(x(k))+g(x(k)) u(k) \\
y(k) & =h(x(k))+J(x(k)) u(k)
\end{aligned}
$$

where $f, g, h, J$ are smooth maps and $f(x) \in \mathfrak{R}^{n}, g(x) \in$ $\mathfrak{R}^{n \times m}, h(x) \in \mathfrak{R}^{m}, J(x) \in \mathfrak{R}^{m \times m}, x \in \mathfrak{R}^{n}, u \in \mathfrak{R}^{m}$.

The characterization for passive systems to use will be the particularization of conditions (5)-(7) for $s(y, u)=$ $y^{T} u$ and the dynamics (13)-(14). Then, conditions (5)(7) takes the following form (Byrnes and Lin, 1993):

$$
\begin{aligned}
& V(f(x))-V(x)=-l^{T}(x) l(x)-m^{T}(x) m(x) \\
& \left.\frac{\partial V(\alpha)}{\partial \alpha}\right|_{\alpha=f(x)} g(x)+2 l^{T}(x) k(x)=h^{T}(x) \\
& \left.g^{T}(x) \frac{\partial^{2} V(\alpha)}{\partial \alpha^{2}}\right|_{\alpha=f(x)} g(x)=J^{T}+J(x)- \\
& -2 k^{T}(x) k(x)
\end{aligned}
$$

Lemma 9. If system (13)-(14) is passive and its zero dynamics is such that $\mathscr{Z}^{*}$ does not contain straight lines of the form $\{(\hat{x}, \lambda \hat{u}), \lambda \in \mathfrak{R}\}$, with $\mathscr{Z}^{*}$ as defined in Section (3), then $J(x)$ is invertible $\forall x$.

Proof Let $\bar{x}$ be such that $J(\bar{x})$ is not invertible. Let us take $\bar{u}(\bar{x})$ such that $\bar{u}(\bar{x}) \in \operatorname{Ker} J(\bar{x})$. Since the system is passive

$$
V(f(x)+g(x) u)-V(x) \leq y^{T} u, \forall(x, u)
$$

Taking into account (18) and the positive definiteness of $V$,

$$
-V(x) \leq[h(x)+J(x) u(x)]^{T} u(x)
$$

If $h^{T}(\bar{x}) \bar{u}(\bar{x}) \neq 0$, defining

$$
u_{0}=-\frac{\lambda V(\bar{x})}{h^{T}(\bar{x}) \bar{u}(\bar{x})},
$$

and restricting inequality (19) to $\bar{x}, u_{0}$, yields

$$
-V(\bar{x}) \leq-\lambda V(\bar{x}), \forall \lambda \in \Re
$$

Thus, $h^{T}(\bar{x}) \bar{u}(\bar{x})=0$, in other words, $\{(\bar{x}, \lambda \bar{u}(\bar{x})), \lambda \in$ $\mathfrak{R}\} \subset \mathscr{Z}^{*}$. Since this is a contradiction with the hypothesis, $J(x)$ must be invertible.

Remark 10. Note that the invertibility of $J(x)$ is equivalent to the fact that system (13)-(14) has relative degree zero.
Theorem 11. Suppose $h(0)=0$, and there exists a $\mathscr{C}^{2}$ storage function $V$, which is positive definite, $V(0)=$ 0 and $V(f(x)+g(x) u)$ is quadratic in $u, \forall f, \forall g$. Then, system (13)-(14) is locally feedback equivalent to a passive system with $V$ as storage function if and only if (13)-(14) has locally relative degree 0 and its zero dynamics is locally passive in a neighbourhood of $x=0$.

Proof (Necessity): If system (13)-(14) is feedback passive, there exists a control $u=\alpha(x)+\beta(x) v$ such that the feedback system

$$
\begin{aligned}
x(k+1) & =\bar{f}(x(k))+\bar{g}(x(k)) v(k) \\
y(k) & =\bar{h}(x(k))+\bar{J}(x(k)) v(k)
\end{aligned}
$$

is passive, with $\bar{f}(x)=f(x)+g(x) \alpha(x), \bar{g}(x)=$ $g(x) \beta(x), \bar{h}(x)=h(x)+J(x) \alpha(x), \bar{J}(x)=J(x) \beta(x)$. Therefore, the transformed system has relative degree zero and passive zero dynamics, see Theorem (8), Lemma (9) and Remark (10).

(Sufficiency): It will be shown that if system (13)(14) has relative degree 0 and passive zero dynamics, it is feedback passive, i.e., there exists a control $u=$ $\alpha(x)+\beta(x) v$, such that the feedback system $x(k+$ 1) $=\bar{f}(x(k))+\bar{g}(x(k)) v(k)$ fulfils

$$
V(\bar{f}(x)+\bar{g}(x) v)-V(x) \leq(\bar{h}(x)+\bar{J}(x) v)^{T} v
$$

If the system relative degree is zero, $J(x)$ is invertible, then

$$
J^{-1}(x)=\left(\frac{\partial}{\partial u} y(x, u)\right)^{-1}
$$

is well defined. It is chosen

$$
u(k)=u^{*}(k)+J^{-1}(x) v(k)
$$

with $u^{*}$ such that $y\left(x, u^{*}\right)=0$, i.e., $u^{*}=-J^{-1}(x) h(x)$. System (13)-(14) with (21) yields to

$$
\begin{aligned}
x(k+1) & =f^{*}(x(k))+g^{*}(x(k)) v(k), \\
y(k) & =v(x(k)),
\end{aligned}
$$

where $f^{*}(x)=f(x)-g(x) J^{-1}(x) h(x)$ represents the zero dynamics of the original system and $g^{*}(x)=$ $g(x) J^{-1}(x)$. Now, a new input control and a new output are defined

$$
y(k)=v(k):=\bar{h}(x(k))+\bar{J}(x(k)) w(k)
$$

Then, the new system dynamics is given by

$$
\begin{aligned}
x(k+1) & =f^{*}(x(k))+g^{*}(x(k)) \bar{h}(x(k))+ \\
& +g^{*}(x(k)) \bar{J}(x) w(k) \\
y(k) & =\bar{h}(x(k))+\bar{J}(x) w(k)
\end{aligned}
$$

It is defined, 


$$
\begin{aligned}
& \bar{J}(x)=\left(\left.\frac{1}{2} g^{*^{T}} \frac{\partial^{2} V}{\partial z^{2}}\right|_{z=f^{*}(x)} g^{*}(x)\right)^{-1} \\
& \bar{h}(x)=-\bar{J}(x)\left(\left.\frac{\partial V}{\partial z}\right|_{z=f^{*}(x)} g^{*}(x)\right)^{T}
\end{aligned}
$$

System (24) with (25) and (26) will be shown to be passive with a $\mathscr{C}^{2}$ storage function $V$. Since $V\left(f^{*}(x)+\right.$ $\left.g^{*}(x) u\right)$ is quadratic in $u$, using the Taylor expansion formula, it follows that

$$
\begin{aligned}
& V\left(f^{*}(x)+g^{*}(x) \bar{h}(x)\right)= \\
= & V\left(f^{*}(x)\right)+\left.\frac{\partial V}{\partial z}\right|_{z=f^{*}(x)} g^{*}(x) \bar{h}(x)+ \\
+ & \left.\frac{1}{2} \bar{h}^{T}(x) g^{*^{T}}(x) \frac{\partial^{2} V}{\partial z^{2}}\right|_{z=f^{*}(x)} g^{*}(x) \bar{h}(x)
\end{aligned}
$$

Substracting $V(x)$ in both sides of (27) and considering that the zero dynamics of (13)-(14) is passive,

$$
\begin{aligned}
& V\left(f^{*}(x)+g^{*}(x) \bar{h}(x)\right)-V(x)= \\
= & -[l(x)+k(x) \bar{h}(x)]^{T}[l(x)+k(x) \bar{h}(x)]- \\
- & m^{T}(x) m(x)+\left.\frac{\partial V}{\partial z}\right|_{z=f^{*}(x)} g^{*}(x) \bar{h}(x)+ \\
+ & \left.\frac{1}{2} \bar{h}^{T}(x) g^{*^{T}}(x) \frac{\partial^{2} V}{\partial z^{2}}\right|_{z=f^{*}(x)} g^{*}(x) \bar{h}(x)
\end{aligned}
$$

Differentiating both sides of (28) with respect to $\bar{h}(x)$, and multiplying the result by $\bar{J}$, in addition to use (25) and (26), the passivity condition (16) for system (24) follows, that is

$$
\begin{gathered}
\left.\frac{\partial V}{\partial z}\right|_{z=f^{*}(x)+g^{*}(x) \bar{h}(x)} g^{*}(x) \bar{J}(x)= \\
=\bar{h}^{T}(x)-2\left[l^{T}(x)+\bar{h}^{T}(x) k^{T}(x)\right] k(x) \bar{J}(x)
\end{gathered}
$$

Taking the second-order derivative with respect to $\bar{h}(x)$ in both sides of (28) and multiplying both sides of the result from the left by $\bar{J}^{T}(x)$ and from the right by $\bar{J}(x)$, using (25) and supposing $J$ to be symmetric, one yields to

$$
\begin{aligned}
& {\left.\left[g^{*}(x) \bar{J}(x)\right]^{T} \frac{\partial^{2} V}{\partial \alpha^{2}}\right|_{\alpha=f^{*}+g^{*} \bar{h}}\left[g^{*}(x) \bar{J}(x)\right]=} \\
= & \bar{J}^{T}(x)+\bar{J}(x)-2 \bar{J}^{T}(x) k^{T}(x) k(x) \bar{J}(x),
\end{aligned}
$$

which is the passivity condition (17) for system (24). For the passivity conditions of system (24), the equivalent of function $l(x)$ is $l(x)+k(x) \bar{h}(x)$, and the equivalent of function $k(x)$ is $k(x) \bar{J}(x)$.

Besides, using (25) and (26) on (27)

$$
V\left(f^{*}(x)+g^{*}(x) \bar{h}(x)\right)=V\left(f^{*}(x)\right)-
$$

$$
-\bar{h}^{T}\left(\bar{J}^{-1}\right)^{T}(x) \bar{h}(x)+\bar{h}^{T} \bar{J}^{-1}(x) \bar{h}(x)=V\left(f^{*}(x)\right)
$$

Taking into account that the original system has passive zero dynamics, i.e., $V\left(f^{*}(x)\right) \leq V(x)$, it can be written

$$
\begin{aligned}
V\left(f^{*}(x)\right) & =V(x)- \\
& -[l(x)+k(x) \bar{h}(x)]^{T}[l(x)+k(x) \bar{h}(x)]- \\
& -m^{T}(x) m(x),
\end{aligned}
$$

then,

$$
\begin{aligned}
& V\left(f^{*}(x)+g^{*}(x) \bar{h}(x)\right)-V(x)= \\
= & -[l(x)+k(x) \bar{h}(x)]^{T}[l(x)+k(x) \bar{h}(x)]- \\
- & m^{T}(x) m(x),
\end{aligned}
$$

which is the passivity condition (15) for the system (24). In conclusion, system (13)-(14) with the passifying control law (23) is passive.

Remark 12. The passifying control given is the same that the control which renders a system lossless in (Byrnes and Lin, 1994).

\section{EXAMPLE}

Let the system, extracted from (Sira-Ramírez, 1991),

$$
\begin{aligned}
& x_{1}(k+1)=\left(x_{1}^{2}(k)+x_{2}^{2}(k)+u(k)\right) \cos \left(x_{2}(k)\right) \\
& x_{2}(k+1)=\left(x_{1}^{2}(k)+x_{2}^{2}(k)+u(k)\right) \sin \left(x_{2}(k)\right) \\
& y(k)=\left(x_{1}^{2}(k)+x_{2}^{2}(k)\right)+J(x(k)) u(k),
\end{aligned}
$$

The control which renders the output to zero is $u^{*}=$ $-J^{-1}(x)\left(x_{1}^{2}+x_{2}^{2}\right)$. Control $u^{*}$ substituted in (32) gives the zero dynamics of the system. It is chosen $J=$ $\frac{1}{x_{1}^{2}+x_{2}^{2}-R^{2}}$ with $R \neq 0$ a constant.

Considering $V=\frac{1}{2}\left(x_{1}^{2}+x_{2}^{2}\right)$, as storage function, and $s=y^{T} u$ as supply function, the system is not passive, in fact, with $u=0$ the origin is unstable. However, the system can be rendered passive by means of a static state feedback control law, see Theorem (11), due to the fact that $J(x)$ is invertible and the zero dynamics of system (32) is passive. Therefore, the passifying control scheme proposed (21)-(26) is applied to (32).

The passified system has been simulated for $x_{0}=$ $[0.01,-1], R=0.5$. It is chosen $w(k)=-y(k)$, as it is proposed in (Lin and Byrnes, 1995), this control locally asymptotically stabilizes a passive discrete-time system. In this case, $w(k)=-[\bar{J}(x(k))+1]^{-1} \bar{h}(x(k))$. The system response is shown in Figure (1). The states and the output are stabilized to the origin $x=$ $(0,0)^{T}, y=0$. 

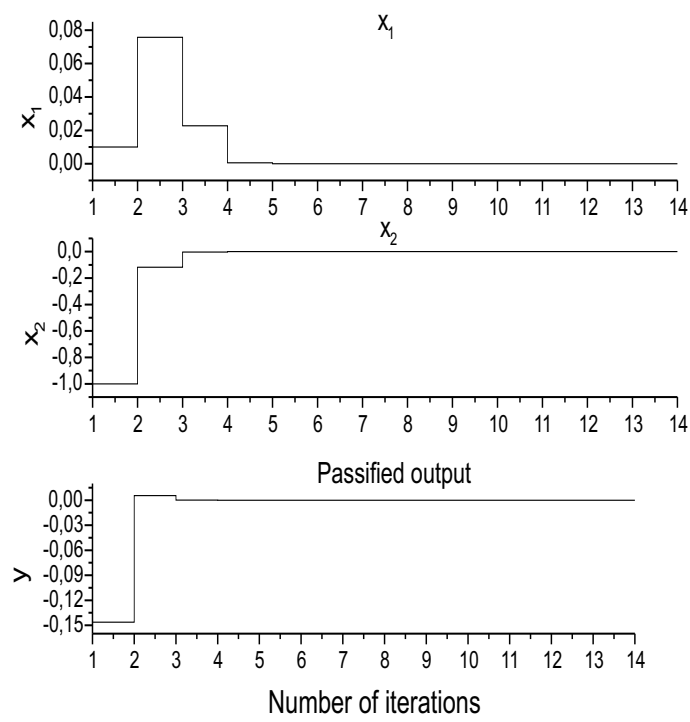

Fig. 1. Passified dynamics for system (32)

\section{CONCLUSIONS}

Multi-input multi-output nonlinear discrete-time dissipative systems have been studied, actually dissipative systems with supply and storage functions satisfying $s(h(x, u), u), V(f(x, u))$ quadratic in $u$. The characterization of this class of dissipative systems has been proposed. In addition, the properties of the relative degree and zero dynamics of passive discretetime systems have been used to prove the solution to the passification problem in nonlinear discrete-time systems affine in the control input. The passification methodology proposed have been validated by means of an example.

There is a great variety of dissipativity-related problems remaining unsolved in the discrete-time setting. New ways of treating dissipativity concepts are needed to be explored. Concerning the results presented here, it should be desirable to give dissipativity conditions without the restriction of $V(f(x, u))$ and $s(h(x, u), u)$ to be quadratic in $u$. This will be a way to give passification conditions for more general systems.

Feedback dissipativity is needed to be solved in order to give desirable energy-like properties to systems whose output does not depend on the input. Analysis of the relative degree and the zero dynamics of dissipative systems would be required, or proposing new approaches to treat dissipativity-related concepts.

\section{REFERENCES}

Byrnes, C.I., A. Isidori and J.C. Willems (1991). Passivity, feedback equivalence, and the global stabilization of minimum phase nonlinear systems. IEEE Trans. on Aut. Control 36, 1228-1240.

Byrnes, C.I. and W. Lin (1993). Discrete-time lossless systems, feedback equivalence, and passivity. Proc. 32nd Conf. on Decision and Control pp. 1775-1781.
Byrnes, C.I. and W. Lin (1994). Losslessness, feedback equivalence, and the global stabilization of discrete-time nonlinear systems. IEEE Trans. on Aut. Control 39(1), 83-98.

Goodwin, G.C. and K.S. Sin (1984). Adaptive Filtering Prediction and Control. Prentice-Hall.

Hill, D.J. and P. Moylan (1980). Dissipative dynamical systems: basic input-output and state properties. Journal of the Franklin Institute.

Hitz, L. and B.D.O. Anderson (1969). Discrete positive-real functions and their application to system stability. Proceedings of IEE 116, 153155.

Lin, W. (1995). Feedback stabilization of general nonlinear control systems: a passive system approach. Systems and Control Letters 25, 41-52.

Lin, W. and C.I. Byrnes (1995). Passivity and absolute stabilization of a class of discrete-time nonlinear systems. Automatica 31(2), 263-267.

Monaco, S. and D. Normand-Cyrot (1997). On the conditions of passivity and losslessness in discrete time. Proc. European Control Conference.

Sengör, N.S. (1995). Energy related concepts in nonlinear systems. Ph.D. Thesis. Instanbul Technical University, Institute of Science and Technology.

Sira-Ramírez, H. (1991). Non-linear discrete variable structure systems in quasi-sliding mode. International Journal of Control 54(5), 1171-1187. 